\title{
Hyperferritinemia in patients with nonalcoholic fatty liver disease
}

Raffaelle K. Barros ${ }^{1}$, Helma Pinchemel Cotrim ${ }^{1 *}$, Carla H. Daltro ${ }^{1}$, Yanaihara A. Oliveira ${ }^{1}$

${ }^{1}$ Medicine and Health Graduate Program (PPgMS), Study Group on Nonalcoholic Steatohepatitis, Faculdade de Medicina da Bahia, Universidade Federal da Bahia, Salvador, BA, Brazill

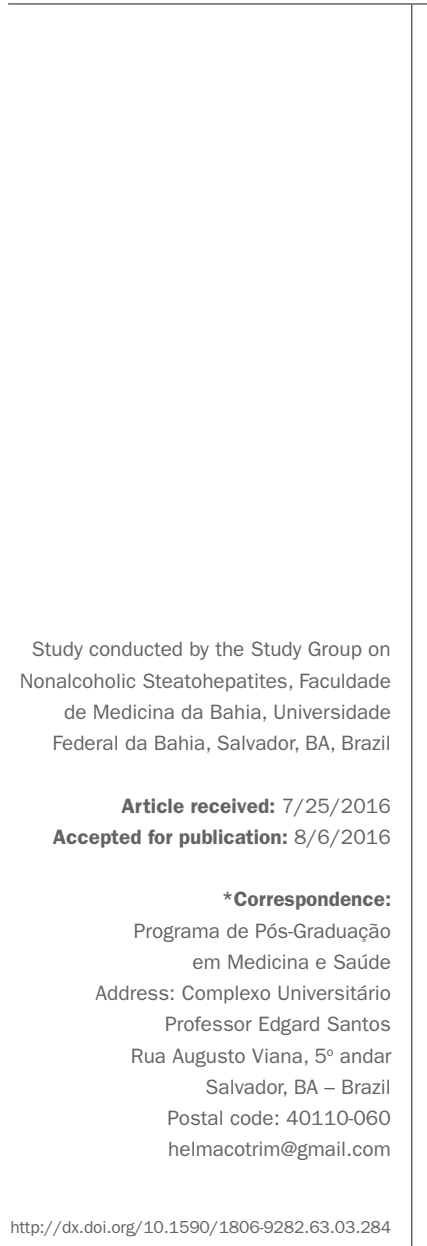

\section{SUMMARY}

Objective: In liver diseases, hyperferritinemia (HYF) is related to injured cells in acquired and genetic conditions with or without iron overload. It is frequent in patients with nonalcoholic fatty liver disease (NAFLD), in which it is necessary to define the mean of HYF to establish the better approach for them. The present study evaluated the significance of elevated ferritin in patients with NAFLD and steatohepatitis (NASH).

Method: The review was performed using search instruments of indexed scientific material, including MEDLINE (by PubMed), Web of Science, IBECS and LILACS, to identify articles published in Portuguese, English and Spanish, from 2005 to May, 2016. Studies eligible included place and year of publication, diagnose criteria to NAFLD, specifications of serum ferritin measurements and/or liver histopathologic study. Exclusion criteria included studies with patients with alcohol consumption $\geq 20 \mathrm{~g}$ /day and other liver diseases.

Results: A total of 11 from 30 articles were selected. It included 3,564 patients and they were cross-sectional, retrospective, case series and case-control. The result's analyses showed in 10 of these studies a relationship between ferritin elevated serum levels and NAFLD/NASH with and without fibrosis and insulin resistance. Conclusion: Hyperferritinemia in patients with NAFLD/NASH is associated more frequently with hepatocellular injury than hemochromatosis. These data suggest the relevance to evaluate carefully HYF in patients with NAFLD/NASH to establish appropriate clinical approach.

Keywords: nonalcoholic fatty liver disease, steatosis, steatohepatitis, hyperferritinemia.

\section{INTRODUCTION}

An association between serum ferritin levels and severity of hepatocellular aggression has been observed in patients with nonalcoholic fatty liver disease (NAFLD) and metabolic syndrome. ${ }^{1,2}$

NAFLD is the most common cause of liver disease today. The spectrum includes steatosis and steatohepatitis (nonalcoholic steatohepatitis, NASH) with increased potential for fibrosis, cirrhosis and hepatocellular carcinoma, and is often associated with increased cardiovascular risk. It is also the hepatic manifestation of metabolic syndrome (MetS), with insulin resistance (IR) as a determining factor in its pathophysiology. ${ }^{3}$

NAFLD has a pathophysiological relationship with hyperinsulinemia, proatherogenic state, and changes in iron metabolism evidenced by hyperferritinemia (HPF) and hemochromatosis, ${ }^{3-5}$ which may have multiple meanings in these patients, since iron accumulation triggers formation of free radicals and damage to cellular function. ${ }^{6}$ In addition, there is iron influence on glucose metabolism, acting as a modulator of insulin secretion by

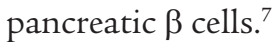

The study of iron metabolism through ferritin dosages, total iron binding capacity and transferrin saturation index is based, initially, on easily investigated serum dosages. Serum dosages may lead to exams or procedures aimed at establishing adequate therapeutic plans and prognosis, avoiding invasive procedures without relevant indication.

Ferritin is an intracellular protein present in all cells involved in iron metabolism, being responsible for the stock of this mineral. It is also an acute phase protein. ${ }^{8,9}$ 
Normal values vary according to ethnicity, age and gender. However, values above $200 \mathrm{mg} / \mathrm{dL}$ for women and 300 $\mathrm{mg} / \mathrm{dL}$ for men have been considered as HPF.

Elevation of serum ferritin may occur in hereditary hemochromatosis, inflammation, liver disease caused by hepatitis B and C virus, and alcoholic liver disease..$^{9-11}$ Our literature review assessed HPF in NAFLD, considering that it can have multiple meanings and that correct interpretation is necessary to guide appropriate clinical conducts for patients with this type of liver disease.

\section{Method}

Articles published in Portuguese, English and Spanish in the last 10 years, until May 2016, were selected from sources such as MEDLINE (PubMed), Scielo and LILACS databases. The following keywords were used in the search for articles: nonalcoholic fatty liver disease, steatosis, steatohepatitis and hyperferritinemia. The articles were eligible when they informed date and place of publication, diagnostic criteria for NAFLD, serum ferritin dosage specifications and histopathological study of the liver. Articles that included patients with a history of alcohol intake greater than $20 \mathrm{~g} /$ day and patients with other chronic liver diseases, such as those caused by alcohol consumption, hepatitis B and C virus, autoimmune hepatitis, and hereditary hemochromatosis, were excluded.

\section{Results}

\section{Analysis of selected articles}

We selected 11 of 30 articles, including cross-sectional, retrospective, case series and case-control studies. Nineteen articles were excluded because they evaluated patients with other liver diseases, or because they did not mention the method for the diagnosis of NAFLD or serum ferritin dosage specifications. Most of the selected studies were conducted in European countries, with samples varying from 37 to 1,014 patients, totaling 3,564 participants. In 10 articles, there was a correlation between ferritin changes and NAFLD/NASH with and without hepatic fibrosis, and insulin resistance. Table 1 shows the main studies.

A correlation between HPF and liver changes in patients with NAFLD/NASH was assessed in these studies. Yoneda et al. ${ }^{12}$ evaluated serum ferritin dosages, the presence of HFE gene mutations, and insulin resistance in 86 Japanese patients and 20 controls. Study participants had NAFLD on histopathological examination of the liver (24 patients with steatosis and 62 patients with NASH). Higher HPF levels were observed in patients with NASH included in those who did not present HFE gene mutation. An association between serum ferritin levels and presence of insu- lin resistance was also found. This study chose individuals with well-established diagnosis of NASH through the histological evaluation performed based on Brunt et al. criteria. ${ }^{13}$ It is important to highlight that the diagnosis of obesity in Japan is considered a body mass index (BMI) $\geq 25 \mathrm{~kg} / \mathrm{m}^{2}$. Mean serum ferritin (in $\mathrm{ng} / \mathrm{dL}$ ) differed between the groups with hepatic steatosis and NASH (164 +/- 95.5 vs. $278.6+/-156.3, \mathrm{p}=0.006)$ as well as in the comparison between healthy individuals, patients with steatosis and patients with NASH $(\mathrm{p}<0.001)$. To assess the accuracy of ferritin levels in NASH, the ideal cutoff point was $196 \mathrm{ng} /$ $\mathrm{mL}$ (95CI $0.596-0.856, \mathrm{p}=0.005)$, with sensitivity of $64.2 \%$, specificity of $76.5 \%$, positive predictive value of $88.9 \%$ and negative predictive value of $43.1 \%$. The only significant correlation while investigating IR was between HPF and HOMA-IR ( $r=0.240, \mathrm{p}=0.0487$ ). They concluded, therefore, that iron overload, IR and oxidative DNA damage correlate in individuals with NASH, and that HPF was the distinguishing characteristic among patients with NASH, representing the most frequent marker of hepatocellular aggression regardless of the presence of mutation in the HFE gene.

Kowdley et al. ${ }^{8}$ observed that HPF is common in patients with NAFLD, and it also increases in inflammatory processes or with increased iron stocks. They studied 628 patients with NAFLD, evaluating serum levels of ferritin. Increases above 1.5 times the upper limit in this method were associated with males, with decreased platelet count and increased transaminases, and independently with advanced hepatic fibrosis (OR 1.66, 95CI 1.05-2.62, $\mathrm{p}=0.028$ ) and increased NAS (NAFLD activity score) (OR 1.99, 95CI 1.06-3.75, $\mathrm{p}=0.033$ ). They observed increased NAS score in subjects without hepatic iron deposition, and suggested that ferritin may be useful in identifying NAFLD in patients at risk for NASH and advanced fibrosis.

Manousou et al., ${ }^{11}$ in search for a diagnostic method for NASH or method of patient selection for liver biopsy, evaluated the relation between high ferritin levels and MetS characteristics versus the presence of inflammation or fibrosis in patients with NAFLD. They studied 111 patients and classified NAFLD in liver biopsy specimens using the Kleiner scoring system. ${ }^{11}$ With a median age of 52.6 years, they included individuals of both sexes and patients with other comorbidities. There was a difference between the steatosis and NASH groups for serum ferritin $(\mathrm{p}=0.0001)$, diabetes mellitus (DM) ( $p=0.001), B M I(p=0.0001)$ and aspartate transaminase $(\mathrm{p}=0.002)$ in this sample. The authors observed that serum ferritin is strongly associated with portal $(\mathrm{p}=0.043)$ and lobular $(\mathrm{p}=0.009)$ inflammation, with sensitivity of $91 \%$ and specificity of $70 \%$ to identify the presence of inflammation or fibrosis in patients with 
TABLE 1 Studies evaluating the relation between hyperferritinemia and NAFLD/NASH.

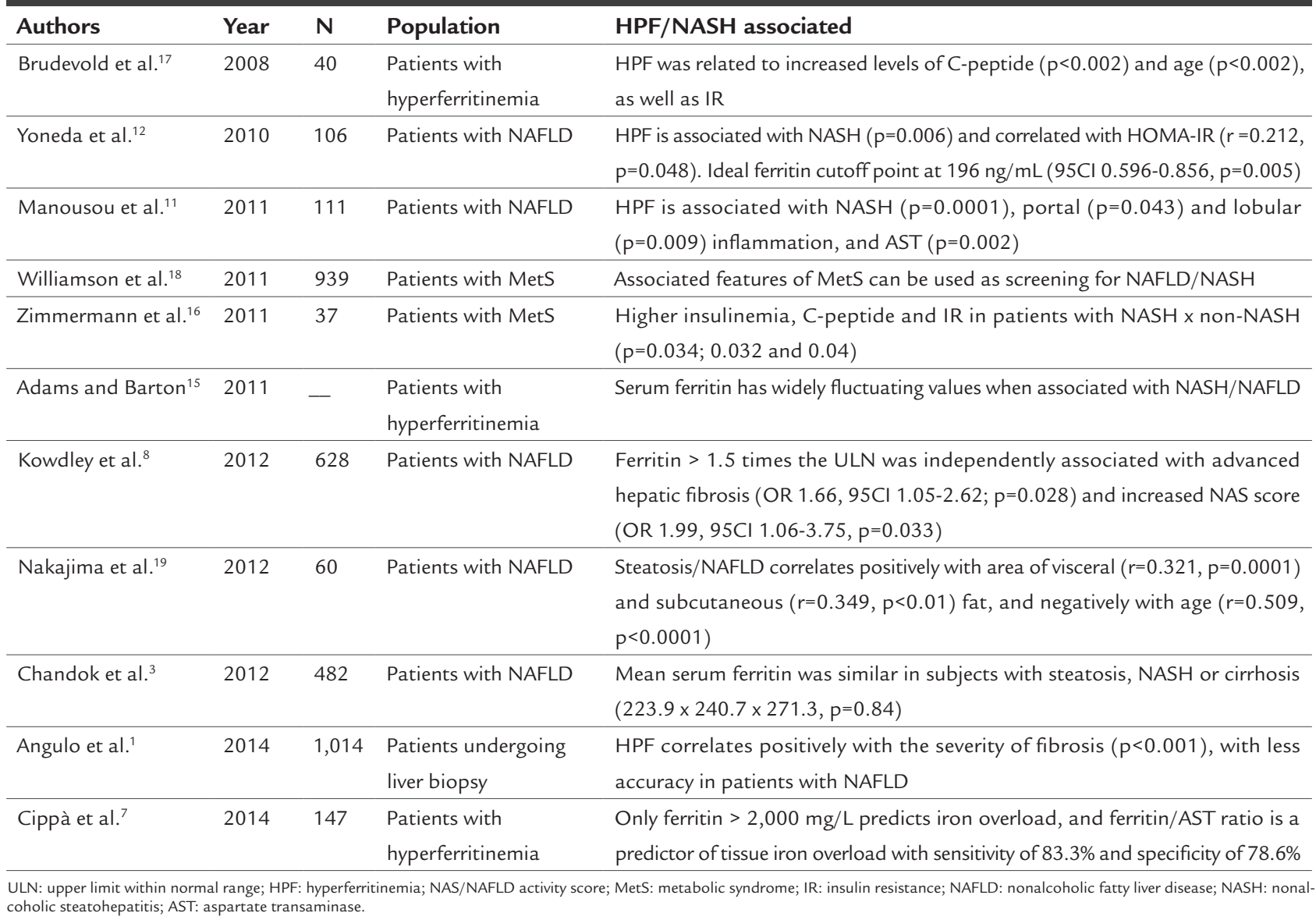

NAFLD. It is therefore a marker that allows to discriminate patients who must undergo liver biopsy, pointing out any association with inflammation and fibrosis.

Angulo et al. ${ }^{1}$ assessed the accuracy of serum ferritin and its association with noninvasive scores (NAFLD fibrosis score, BARD, APRI and FIB-4) to determine the presence and severity of liver fibrosis. They retrospectively studied 1,014 patients undergoing liver biopsy and concluded that serum ferritin levels identify fibrosis with $16-41 \%$ sensitivity and $70-92 \%$ specificity, and correlate positively with fibrosis severity $(\mathrm{p}<0.001)$. However, they also observed that when applied alone, ferritin is less accurate in patients with NAFLD. In addition, the accuracy of noninvasive scores to indicate patients with advanced fibrosis did not increase when added to HPF. They concluded that serum ferritin cannot be used to diagnose the presence or severity of hepatic fibrosis, although normal or slightly increased ferritin may exclude such diagnostic possibility.

Adams e Barton ${ }^{14}$ noted that HPF is typically stable over time in patients with inherited clinical conditions such as hemochromatosis, hereditary hyperferritinemia-cataract syndrome and in certain racial groups, whereas serum ferritin levels are widely fluctuating when induced by alcohol or associated with NASH/NAFLD.

Chandok et al. ${ }^{15}$ studied 482 patients, but only 108 of these subjects underwent histological or radiological investigation and had liver abnormalities compatible with NAFLD. They found that AST/ALT ratio, DM, splenomegaly and age were predictors of the NAFLD stage. Mean serum ferritin was similar in subjects with steatosis, NASH or cirrhosis (223.9 x $240.7 \times 271.3, \mathrm{p}=0.84)$, suggesting that HPF magnitude does not allow conclusions about the stage of the liver disease, but is simply higher in most patients with NAFLD.

Cippà et al. ${ }^{7}$ analyzed 147 patients with HPF who underwent liver biopsy due to suspicion of liver disease. These authors suggest that HPF is only a predictor of hemochromatosis in cases above $2,000 \mathrm{mg} / \mathrm{L}$. They also suggest that the elevated ferritin/AST ratio can be a predictor of hepatic iron overload. The sensitivity for these tests was $83.3 \%$ and the specificity of $78.6 \%$. 
Some studies have suggested associations between MetS and NAFLD/NASH. They include the work of Zimmermann et al., ${ }^{16}$ who studied the lipid, glycemic and iron metabolism profiles of 37 individuals with MetS, 25 of whom were diagnosed with NASH. The authors observed that NASH patients had higher insulinemia, serum C-peptide and IR levels compared to non-NASH ( $\mathrm{p}=0.034$, $\mathrm{p}=0.032$ and $\mathrm{p}=0.04$ ), whereas NASH with grade 2 and 3 steatosis had higher LDL-cholesterol compared to patients with grade 1 steatosis $(p=0.002)$, non-NASH $(\mathrm{p}=0.03)$ and healthy controls $(\mathrm{p}=0.001)$. These results suggest the relation of NASH with inflammation, altered carbohydrate metabolism and atherogenic profile.

Brudevold et al. ${ }^{17}$ assessed the relationship between MetS and HPF in 40 patients consecutively referred for investigation of this disorder in 2001. Among the 29 subjects who underwent hepatic biopsy, 19 had steatosis and four had iron overload; $52 \%$ met criteria for MetS according to IDF criteria. They observed that HPF was related to increased levels of $\mathrm{C}$-peptide $(\mathrm{p}<0.002)$ and age $(\mathrm{p}<0.002)$, as well as to IR.

Williamson et al., ${ }^{18}$ studying DM2 as a risk factor for the development of hepatic steatosis and NAFLD in a cohort of 939 participants aged 61-76 years in Edinburgh, state that the combination of characteristics of MetS can be used as screening for NAFLD/NASH. Randomized through ultrasonography, they found hepatic steatosis in $56.9 \%$ and NAFLD in $42.6 \%$. Independent predictors included: BMI, decreased duration of DM, glycosylated hemoglobin, triglycerides and use of metformin.

Lastly, Nakajima et al. ${ }^{19}$ studied 60 patients diagnosed with NAFLD. Based on the assumption that hepatic steatosis is a result of excess visceral fat and represents the starting point in the pathogenesis of NASH, they demonstrated that the degree of steatosis/NAFLD correlates positively with the area of visceral $(\mathrm{r}=0.321, \mathrm{p}=0.0001)$ and subcutaneous $(\mathrm{r}=0.349, \mathrm{p}=<0.01)$ fat, and negatively with age $(\mathrm{r}=0.509, \mathrm{p}<0.0001)$.

\section{Discussion}

In the present review of the literature we observed different selection criteria and methods among the articles, which makes it difficult to compare the results.

HPF has been correlated in many articles with the highest risk of liver fibrosis, especially in patients with NAFLD. Hepatocellular aggression is the most frequent cause of increased HPF in patients with NAFLD/NASH. It is clear that metabolic alterations such as hyperinsulinemia, dysglycemia, dyslipidemia and insulin resistance aggravate the clinical picture and progression of NAFLD. These studies stress the necessary care in interpreting HPF.

Ferritin is an acute phase protein that increases in the presence of systemic inflammation, commonly related to obesity, diabetes and metabolic syndrome. It plays an important role in the storage of intracellular iron and is present in several cell types. Serum levels are often high in individuals with chronic and daily consumption of alcoholic beverages and other chronic liver diseases, such as hepatitis caused by viruses B and C. Increased ferritin may be observed in acute or drug-induced hepatitis, neoplastic, hematological, and other diseases. ${ }^{9-11,19-22}$

Serum ferritin dosage is a simple, low-cost laboratory test, which may be a good marker of hepatocellular aggression and liver fibrosis in the selection of patients who will actually require hepatic biopsy.

We have observed in several studies included in this review that ferritin may be an independent predictor of inflammation and liver fibrosis, and may contribute to the decision to perform biopsy in individuals clinically diagnosed with NAFLD. ${ }^{23-27}$

It has been proposed that the sum of the HPF, BMI, DM and AST variables can predict the risk of NASH with sensitivity of $92 \%$ and specificity of $80 \%$ as well as severe fibrosis. ${ }^{23,27}$ Nevertheless, it was observed that noninvasive scores have diagnostic accuracy to distinguish between the presence or absence of advanced fibrosis, which did not increase with the addition of ferritin as a variable. ${ }^{1}$

Cippà et al. considered HPF as a predictor of hemochromatosis only for values above $2,000 \mathrm{mg} / \mathrm{L} .{ }^{23}$ Angulo et al. showed that ferritin correlates positively with fibrosis severity but cannot be used to diagnose the presence or severity of liver fibrosis. ${ }^{1}$

The manifestations of MetS are influenced by visceral adiposity (VA), but unlike hyperinsulinemia and IR, VA does not determine greater severity of liver damage in patients with NAFLD. In contrast, adiponectin, an insulin-sensitizing adipokine characteristically decreased in obesity, is an independent risk factor for NAFLD. Nevertheless, the option of screening and monitoring of NASH and hepatic fibrosis should not be differentiated in lean individuals. ${ }^{12,27,28}$

There is controversy regarding the results of phlebotomy to eliminate excess iron. Some believe that there is no improvement in steatosis, liver damage or IR. ${ }^{29} \mathrm{In}$ another line of reasoning, it is suggested that iron depletion allows a more relevant improvement of liver enzyme levels and histological changes present in NAFLD, ${ }^{6,30}$ 
Out of 11 studies evaluated in our review, the only discordant one regarding the interpretation of HPF was that by Chandok et al. ${ }^{3}$ In the article, HPF was similar in patients with steatosis, NASH or cirrhosis. We speculate whether this finding could be justified by the fact that only $19.3 \%$ of the participants in this study were evaluated by liver biopsy and histological analysis. ${ }^{15}$ All other studies agree on the existence of an association between HPF and NASH with severe liver fibrosis.

It has been observed that in patients with NAFLD, insulin resistance is frequently present and associated with HPF, a clinical expression of hepatocellular inflammation. In contrast, less frequent and late iron overload is associated with oxidative DNA damage. ${ }^{16,31}$ Thus, the association between HPF and NASH with severe hepatic fibrosis has implications for the diagnosis and clinical follow-up of patients with NAFLD/NASH. ${ }^{2}$

\section{Conclusion}

Hyperferritinemia in patients with NAFLD/NASH is associated more frequently with hepatocellular aggression than with iron overload. The results of our review suggest the need for greater caution while interpreting serum ferritin elevation in patients with NAFLD/NASH to determine appropriate clinical management.

\section{Conflict of interest}

The authors declare no conflict of interest.

\section{Resumo}

Hiperferritinemia em pacientes com doença hepática gordurosa não alcoólica

Objetivo: A hiperferritinemia (HPF) está associada à agressão hepatocelular nas doenças do fígado e à sobrecarga de ferro, em doenças genéticas e adquiridas. A HPF é frequente em pacientes com doença hepática gordurosa não alcoólica (DHGNA) e é necessário definir seu significado para estabelecer as melhores condutas para esses indivíduos. Esta revisão avaliou o significado da HPF em portadores de DHGNA e esteato-hepatite não alcoólica (EHNA).

Método: A busca de artigos foi realizada através do PubMed (Medline), Web of Science e Lilacs, e foram selecionados aqueles publicados em português, inglês e espanhol de 2005 a maio de 2016. Os artigos foram elegíveis quando informavam data e local da publicação, critérios diagnósticos para DHGNA, especificações das dosagens de ferritina sérica e/ou estudo histopatológico.
Foram excluídos os artigos cujos pacientes relataram ingestão alcoólica $\geq 20 \mathrm{~g} / \mathrm{dia}$ ou eram portadores de outras doenças do fígado.

Resultados: Foram selecionados 11 de 30 artigos, totalizando 3.564 pacientes. Os artigos eram de corte transversal, retrospectivos, série de casos e caso-controles. Em dez artigos, observou-se correlação entre alteração de ferritina e DHGNA/EHNA com e sem fibrose hepática e resistência à insulina.

Conclusão: Hiperferritinemia em pacientes com DHGNA/ EHNA se associa com maior frequência à agressão hepatocelular do que com sobrecarga de ferro hepático. Os resultados da revisão sugerem a necessidade de um maior cuidado na interpretação da elevação da ferritina sérica em pacientes com DHGNA/EHNA para o estabelecimento de condutas clínicas apropriadas.

Palavras-chave: doença hepática gordurosa não alcoólica, esteatose, esteato-hepatite, hiperferritinemia.

\section{References}

1. Angulo P, George J, Day CP, Vanni E, Russell L, De la Cruz AC, et al. Serum ferritin levels lack diagnostic accuracy for liver fibrosis in patients with nonalcoholic fatty liver disease. Clin Gastroenterol Hepatol. 2014; 12(7):11639.

2. Valenti L, Dongiovanni P, Fargion S. Diagnostic and therapeutic implications of the association between ferritin level and severity of nonalcoholic fatty liver disease. World J Gastroenterol. 2012; 18(29):3782-6.

3. Chandok N, Minuk G, Wengiel M, Uhanova J. Serum ferritin levels do not predict the stage of underlying non-alcoholic fatty liver disease. J Gastrointestin Liver Dis. 2012; 21(1):53-8.

4. Ferrannini E. Insulin resistence, iron, and the liver. Lancet. 2000; 355(9222):2181-2.

5. Ferreira VSG, Pernambuco RB, Lopes EP, Morais CN, Rodrigues MC, Arruda $\mathrm{MJ}$, et al. Frequency and risk factors associated with nonalcoholic fatty liver disease in patients with type 2 diabetes mellitus. Arq Bras Endocrinol Metabol. 2010; 54(4):362-8.

6. Adams LA, Crawford DH, Stuart K, House MJ, St Pierre TG, Webb M, et al. The impact of phlebotomy in nonalcoholic fatty liver disease: a prospective, randomized, controlled trial. Hepatology. 2015; 61(5):1555-64.

7. Cippà PE, Boucsein I, Adams H, Krayenbuehl PA. Estimating iron overload in patients with suspected liver disease and elevated serum ferritin. Am J Med. 2014; 127(10):1011.e1-3.

8. Kowdley KV, Belt P, Wilson LA, Yeh MM, Neuschwander-Tetri BA, Chalasani $\mathrm{N}$, et al.; NASH Clinical Research Network. Serum ferritin is an independent predictor of histologic severity and advanced fibrosis in patients with nonalcoholic fatty liver disease. Hepatology. 2012; 55(1):77-85.

9. Cotrim HP. Hyperferritinaemia. UEG Week 2013; 148-51. Available from: https://d1m2qru0mx8r19.cloudfront.net/uegfolga-web/uegf-ep-mat089/ IP060.pdf.

10. Kell DB, Pretorius E. Serum ferritin is an important inflammatory disease marker, as it is mainly a leakage product from damaged cells. Metallomics. 2014; 6(4):748-73.

11. Manousou P, Kalambokis G, Grillo F, Watkins J, Xirouchakis E, Pleguezuelo $\mathrm{M}$, et al. Serum ferritin is a discriminant marker for both fibrosis and inflammation in histologically proven non-alcoholic fatty liver disease patients. Liver Int. 2011; 31(5):730-9.

12. Yoneda M, Nozaki Y, Endo H, Mawatari H, Iida H, Fujita K, et al. Serum ferritin is a clinical biomarker in Japanese patients with nonalcoholic steatohepatitis (NASH) independent of HFE gene mutation. Dig Dis Sci. 2010; 55(3):808-14 
13. Brunt EM, Kleiner DE, Wilson LA, Belt P, Neuschwander-Tetri BA; NASH Clinical Research Network (CRN). Nonalcoholic fatty liver disease (NAFLD) activity score and the histopathologic diagnosis in NAFLD: distinct clinicopathologic meanings. Hepatology. 2011; 53(3):810-20.

14. Aigner E, Weiss G, Datz C. Dysregulation of copper homeostasis in nonalcoholic fatty liver. World J Hepatol. 2015; 7(2):177-88.

15. Adams $\mathrm{P}$, Barton J. A diagnostic approach to hyperferritinemia with a nonelevated transferrin saturation. J Hepatol. 2011; 55(2):453-8.

16. Zimmermann A, Zimmermann T, Schattenberg J, Pöttgen S, Lotz J, Rossmann $\mathrm{H}$, et al. Alterations in lipid, carbohydrate and iron metabolism in patients with non-alcoholic steatohepatitis (NASH) and metabolic syndrome. Eur J Intern Med. 2011; 22(3):305-10.

17. Brudevold R, Hole T, Hammerstrøm J. Hyperferritinemia is associated with insulin resistance and fatty liver in patients without iron overload. PLoS ONE. 2008; 3(10):e3547.

18. Williamson RM, Price JF, Glancy S, Perry, E, Nee LD, Hayes PC, et al. Edinburgh Type 2 Diabetes Study Investigators. Prevalence of and risk factors for hepatic steatosis and nonalcoholic fatty liver disease in people with type 2 diabetes: the Edinburgh type 2 diabetes study. Diabetes Care. 2011; 34(5):1139-44

19. Nakajima T, Nakashima T, Yamaoka J, Shibuya A, Itoh Y, Yoshikawa T. Age is a negative, and visceral fat accumulation is a contributor to hepatic steatosis, regardless of the progression in non-alcoholic fatty liver disease. J Gastroenterol Hepatol Res. 2012; 1(11):315-9.

20. Bozzini C, Girelli D, Olivieri O, Martinelli N, Bassi A, De Matteis G, et al. Prevalence of body iron excess in the metabolic syndrome. Diabetes Care. 2005; 28(8):2061-3.

21. Bell H, Skinningsrud A, Raknerud N, Try N. Serum ferritin and transferrin saturation in patients with chronic alcoholic and non-alcoholic liver diseases J Intern Med. 1994; 236(3):315-22.
22. Tung BY, Emond MJ, Bronner MP, Raaka SD, Cotler SJ, Kowdley KV. Hepatitis C, iron status, and disease severity: relationship with HFE mutations. Gastroenterology. 2003; 124(2):318-26.

23. Ucar F, Sezer S, Erdogan S, Akyol S, Armutcu F, Akyol O. The relationship between oxidative stress and nonalcoholic fatty liver disease: its effects on the development of nonalcoholic steatohepatits. Redox Rep. 2013; 18(4):127-33.

24. Wieckowska A, Papouchado BG, Li Z, Lopez R, Zein NN, Feldstein AE. Increased hepatic and circulating interleukin-6 levels in human nonalcoholic steatohepatitis. Am J Gastroenterol. 2008; 103(6):1372-9.

25. Estep JM, Baranova A, Hossain N, Elariny H, Ankrah K, Afendy A, et al Expression of cytokine signaling genes in morbidly obese patients with nonalcoholic steatohepatitis and hepatic fibrosis. Obes Surg. 2009; 19(5):617-24

26. Syn WK, Choi SS, Diehl AM. Apoptosis and cytokines in nonalcoholic steatohepatitis. Clin Liver Dis. 2009; 13(4):565-80.

27. Ibrahim MA, Kelleni M, Geddawy A. Nonalcoholic fatty liver disease: current and potential therapies. Life Sci. 2013; 92(2):114-8.

28. Wang Y, Zhou M, Lam KS, Xu A. Protective roles of adiponectin in obesityrelated fatty liver diseases: mechanisms and therapeutic implications. Arq Bras Endocrinol Metab. 2009; 53(2):201-12.

29. Valenti L, Fracanzani AL, Dongiovanni P, Rovida S, Rametta R, Fatta E, et al. A randomized trial of iron depletion in patients with nonalcoholic fatty liver disease and hyperferritinemia. World J Gastroenterol. 2014; 20(11):3002-10.

30. Fernández-Real JM, McClain D, Manco M. Mechanisms linking glucose homeostasis and iron metabolism toward the onset and progression of type 2 diabetes. Diabetes Care. 2015; 38(11):2169-76.

31. Fujita N, Miyachi H, Tanaka H, Takeo M, Nakagawa N, Kobayashi Y, et al Iron overload is associated with hepatic oxidative damage to DNA in nonalcoholic steatohepatitis. Cancer Epidemiol Biomark Prev. 2009; $18(2): 424-32$. 